Special issue of the 3rd International Conference on Computational and Experimental Science and Engineering (ICCESEN 2016)

\title{
A New Mathematical Model for Multisession Exams-Building Assignment
}

\author{
Z. ERGUL* AND Z. KAMisLi OZTURK \\ Anadolu University, Industrial Engineering Department, Eskisehir, Turkey
}

\begin{abstract}
The educational timetabling problem has been extensively investigated in timetabling literature. However, the problem of assigning exams to examination buildings has not been studied intensively by researchers. We were inspired by Open and Distance Education System exams of Anadolu University. Anadolu University Open and Distance Education System, which is used by approximately two millions of students and has more than two millions of graduates, is a well-known institution in Turkey. In this study, we propose a multi-objective mathematical model for multisession exam-building assignment problem. Objective functions of this model are to minimize the distance between consecutive session buildings for a given student, to maximize the number of occupants of buildings in every session and to minimize the variety of booklets for building in every session. Mathematical model has been found inadequate because students-examination building assignment in the Anadolu University Open Education system is a large size real life problem. Starting from this point of view, an order-based multi-objective heuristic algorithm is developed to solve this problem. The solutions obtained by the proposed algorithm are compared with the solution obtained by the mathematical modelling and the current state of the existing system.
\end{abstract}

DOI: 10.12693/APhysPolA.132.1207

PACS/topics: Educational timetabling, Examination-building assignment, Multi objective nonlinear optimization, Mixed Integer Programming

\section{Introduction}

Timetabling problems are a kind of assignment problems that deal with assigning a set of activities to a set of resources and time periods under a set of predetermined constraints. Timetabling problems range from construction of weekly course timetables and/or exam schedules in schools, colleges, and universities through to the timetabling of transport facilities such as buses, trains, or aircrafts [1]. Educational timetabling deals with timetabling at educational institutes. Educational timetabling is an important operational problem in many schools, colleges and universities [2]. Educational timetabling problems can be grouped into two categories; course scheduling and exam scheduling. Most educational institutions must schedule a set of examinations at the end of each session or year [3]. This study deals with exam-room assignment, which is under the head of exam scheduling. Exam-room assignment problems have not been extensively investigated in exam-timetabling literature [4].

In general, real life problems [5-7] have multi objective structure. We focused on the works in literature, which have similar objective functions to our problem and have summed up these works briefly in the following. Kahar and Gendall [8] presented a real-life problem, which is capacitated examination timetabling problem with two new constraints. These constraints are the travel distance for lecturers/invigilators and splitting exams across rooms. They developed a graph coloring based algorithm. Dammak et al. [4] considered the problem of assigning a set of

*corresponding author; e-mail: zergul@anadolu.edu.tr independent (non-conflicting) exams, having a given size, to a set of classrooms, having certain capacities, and formulated the problem as a transportation problem. In addition, they proposed a heuristic procedure known as max-size assignment to solve large scale problems. Sarin et al. [9] presented a methodology that relies on Benders' partitioning for university-timetabling problem. The performance of this methodology was measured by the total distance traveled by the faculty members from their offices in respective departments to the classrooms in which the courses were offered. Ayob and Malik [10] aimed to minimize the amount of students' movement between multiple rooms and suggested a heuristic that can be used to solve the model. Vermuyten et al. [11] presented a two-stage integer programming approach for a university course timetable that aims at minimizing the resulting student flows in the buildings.

In this study, the considered educational institute is Anadolu University Open and Distance Education System (ODES) which is the first and leading institution in Turkey, that offers higher distance education. Anadolu University ODES sets four examinations (two midterms, two final exams) for approximately 1400000 students, who have enrolled in the approximately 51 programs for each full semester. Each examination is held in four sessions on Saturdays and Sundays. In the fall semester, examinations of the first and fifth semesters are held in morning sessions and examinations of the third and seventh semesters are held in afternoon sessions. In the spring semester, examinations of the second and sixth semesters are held in morning sessions and examinations of the fourth and eighth semesters are held in afternoon sessions on Saturdays and Sundays. Each booklet in each session includes number of courses, from 1 to 5 , and each 
course has 20 questions. Students have 30 minutes to answer these questions [12]. Anadolu University ODES uses approximately 9666 examination buildings, 144232 classrooms and 4311445 workers in 98 examination centers for each examination. Students, who were assigned to consecutive sessions, complain about distance between their examination buildings for the same day. Sometimes they cannot reach their second session examination building in the same day because of the distance between two examination buildings. So, this dissatisfaction is the motivation of our study.

In Section 2, we develop a multi objective mixed integer nonlinear mathematical model for examination building assignment for students. Based on the large scale of this real life problem, a heuristic solution algorithm is also proposed. Finally, in Section 3 the conclusions are given.

\section{Solution approaches for multisession exams-building assignment}

\subsection{Mathematical model}

For student-examination building assignment of ODES a multi objective mixed integer nonlinear mathematical model has been developed with the following sets, parameters, decision variables, constraints and objectives.

\subsubsection{Sets}

Students: $I=\{1, \ldots, m\}$

Sessions: $J=\{1, \ldots, 4\}$

Examination buildings: $K, L=\{1, \ldots, p\}$

Booklets: $H=\{1, \ldots, o\}$

2.1.2. Parameters of the problem

$d_{k l}$ : distance matrix (between examination buildings)

$q_{k}$ : capacity of examination buildings

$K T_{h j}$ : booklet-session matrix

$O K_{i h}$ : student-booklet matrix

$M$ : a very big number $(\operatorname{Big} M)$

\subsubsection{Decision variables}

$x_{i j k}=1$ if student $i$ is assigned to examination building $k$ in session $j ; 0$ otherwise.

$y_{i j}=1$ if student $i$ is assigned to session $j ; 0$ otherwise.

$b_{j k}=1$ if session $j$ is assigned to examination building $k ; 0$ otherwise.

$c_{j k}=1$ is the unused capacity of examination building $k$, assigned to session $j$.

$p_{k h}=1$ is the number of booklets $h$ in examination building $k$.

$r_{k h}=1$ if booklet $h$ is assigned to examination building $k ; 0$ otherwise.

\subsubsection{Constraints}

$$
\begin{aligned}
& \sum_{j=1}^{4} y_{i j} \geq 1, \quad \forall i \\
& y_{i j}=\sum_{h=1}^{0} O K_{i h} K T_{h j}, \quad \forall(i, j)
\end{aligned}
$$

$$
\begin{aligned}
& \sum_{k=1}^{p} x_{i j k} \leq 1, \quad \forall(i, j) \\
& \sum_{j=1}^{4} b_{j k} \leq 4, \quad \forall k \\
& \sum_{i=1}^{m} x_{i j k} \leq q_{k}, \quad \forall(j, k) \\
& y_{i j} \leq \sum_{k=1}^{p} x_{i j k} \leq y_{i j} \times M, \quad \forall(i, j) \\
& b_{j k} \leq \sum_{i=1}^{m} x_{i j k} \leq b_{j k} \times M, \quad \forall(j, k) \\
& c_{j k}=b_{j k} \times q_{k}-\sum_{i=1}^{m} x_{i j k}, \quad \forall(j, k) \\
& p_{k h}=\sum_{j=1}^{4} \sum_{i=1}^{m} x_{i j k} \times O K_{i h} \times K T_{h j}, \quad \forall(k, h) \\
& r_{k h} \leq p_{k h} \leq r_{k h} \times M, \quad \forall(k, h) \\
& x_{i j k}, y_{i j}, b_{j k}, r_{k h} \in\{0,1\}, \\
& c_{j k}, p_{k h} \geq 0, \quad \forall(i, j, k, h)
\end{aligned}
$$

Equation (1) ensures that each student is assigned to at least one session. Equation (2) ensures that each student is assigned to a session, according to bookletsession matrix and student-booklet matrix. Equation (3) guarantees that each student is assigned to at most one examination building in each session. Equation (4) ensures that each examination building is assigned to at most four sessions. Equation (5) is a capacity constraint, which guarantees that the number of students is assigned to $k^{t h}$ building is less than or equal to building's capacity. Equations (6), (7), (8), (9) and (10) are the relation constraints between the decision variables. Equation (11) gives the sign constraints.

The scalar objective function, as given in Eq. (15), consists of three terms. The first term, as given in Eq. (12), represents the total distance traveled by students, who have two consecutive sessions during the same day. The second term represents the total unused capacity of examination buildings (Eq. (13)). Finally, Eq. (14) represents the total booklet diversity of examination buildings.

Each of these three objective parameters is required to be minimized. In addition, to obtain a scalar sum of these three objectives, they were normalized.

$$
\begin{aligned}
F_{1} & =\left(\sum_{i=1}^{m} \sum_{k=1}^{p} \sum_{l=1, k \neq l}^{p} x_{i 1 k} x_{i 2 l} d_{k l}\right. \\
& \left.+\sum_{i=1}^{m} \sum_{k=1}^{p} \sum_{l=1, k \neq l}^{p} x_{i 3 k} x_{i 4 l} d_{k l}\right) / \max \left(d_{k l}\right),
\end{aligned}
$$




$$
\begin{aligned}
& F_{2}=\sum_{k=1}^{p} \sum_{j=1}^{4}\left(c_{j k} / q_{k}\right), \\
& F_{3}=\sum_{k=1}^{p} \sum_{h=1}^{0}\left(r_{h k} / o\right),
\end{aligned}
$$

$\min Z=F_{1}+F_{2}+F_{3}$.

Test problems given in Table I were solved by the optimization software GAMS 23.3.3.

TABLE I

Test problems

\begin{tabular}{c|c|c|c|c}
\hline \hline & Test 1 & Test 2 & Test 3 & Test 4 \\
\hline Number of student & 21408 & 257 & 257 & 50 \\
Number of session & 2 & 2 & 2 & 2 \\
No of examination buildings & 62 & 62 & 12 & 5 \\
Number of booklet diversity & 115 & 124 & 124 & 20
\end{tabular}

TABLE II

GAMS results, execution time $t$ and objective function $Z$.

\begin{tabular}{c|c|c|c|c|c|c}
\hline \hline & \multicolumn{2}{|c|}{ Test 2} & \multicolumn{2}{c|}{ Test 3 } & \multicolumn{2}{c}{ Test 4} \\
\hline Solver & $t[\mathrm{~s}]$ & $Z$ & $t[\mathrm{~s}]$ & $Z$ & $t[\mathrm{~s}]$ & $Z$ \\
\hline BARON & - & - & 1489 & 44.342 & 1005 & 1.35 \\
SBB & - & - & 1441 & 44.342 & 62 & 21.6 \\
DICOPT & 514 & 2.228 & 5 & 2.672 & 1 & 1.35
\end{tabular}

GAMS was not able to generate any solution for Test 1 , which represents a medium size problem. As the problem is a nonlinear mixed integer programming problem, we chose suitable solvers of GAMS and the obtained results for the remaining test problems are given in Table II. These results are the best objective function values, obtained when the solvers stop searching for the optimal solution. Thus, it is clear that a heuristic solution approach is required for Anadolu University ODES large dimension (with approximately 1400000 students) problem.

\subsection{Multi-objective heuristics}

Multi objective heuristic algorithm uses additional parameters, which are upper limit of examination building's booklet diversity and lower limit of examination building's fill rate. This algorithm firstly assigns students to examination buildings in morning session and then assigns them to examination buildings in afternoon session, for each day with order-based procedures. The heuristic algorithm aims at assigning students, which have consecutive sessions, to as close as possible examination buildings during a day. For order-based procedure; the number of students are ordered decreasingly, based on diversity of booklets. Examination buildings are ordered decreasingly, based on their capacities and distance between the other buildings. The obtained solutions are evaluated and tried to be improved, according to the diversity of booklet amount of the buildings. To demonstrate the effectiveness of the proposed algorithm, three test problems are solved. The obtained three objective function values (Eq. (12), Eq. (13), Eq. (14)) of the proposed mathematical model and of the heuristic algorithm are given in Table III. It is seen that for minimum total traveled distance $F_{1}$ the heuristic algorithm performs better than mathematical model. However, mathematical model performs better than heuristic algorithm for minimum total unused capacity $F_{2}$ and the total booklet diversity $F_{3}$.

TABLE III

Results obtained from mathematical model, compared to multi objective heuristic algorithm.

\begin{tabular}{c|c|c|c|c|c|c|c|c|c}
\hline \hline & \multicolumn{3}{|c|}{ Test 2 } & \multicolumn{3}{c|}{ Test 3 } & \multicolumn{3}{c}{ Test 4 } \\
\cline { 2 - 9 } & $F_{1}$ & $F_{2}$ & $F_{3}$ & $F_{1}$ & $F_{2}$ & $F_{3}$ & $F_{1}$ & $F_{2}$ & $F_{3}$ \\
\hline Math. model & 0.058 & 0 & 2.169 & 0 & 1.12 & 1.548 & 0 & 0 & 1.35 \\
Multi-obj. heur. & 0 & 11.67 & 3.62 & 0 & 4.087 & 2.856 & 0 & 1 & 3.2
\end{tabular}

TABLE IV

Comparison of results obtained by multi objective heuristic algorithm with the current schedule.

\begin{tabular}{l|c|c|c|c|c|c|c|c}
\hline \hline \multicolumn{2}{|c|}{} & \multicolumn{2}{|c|}{$\begin{array}{c}\text { Performance of distance } \\
\text { criterium }[\mathrm{km}]\end{array}$} & $\begin{array}{c}\text { Performance of diversity } \\
\text { criterium }\end{array}$ & \multicolumn{2}{c}{$\begin{array}{c}\text { Performance of fill rate } \\
\text { criterium [\%] }\end{array}$} \\
\cline { 3 - 9 } \multicolumn{2}{c|}{} & Average & Max & Total & Average & Max & Min & Average \\
\hline \multirow{2}{*}{ Saturday } & Current & 3.317 & 9.546 & 23427.97 & 5.029 & 14 & 60.032 & 94.110 \\
& Heuristic & 0.002 & 1.852 & 15.184 & 3.448 & 5 & 7.732 & 56.118 \\
\hline \multirow{2}{*}{ Sunday } & Current & 3.383 & 9.550 & 25723.240 & 5.222 & 17 & 40.230 & 95.670 \\
& Heuristic & 0.001 & 0.520 & 9.713 & 3.509 & 5 & 7.732 & 56.118
\end{tabular}


After these tests, a real examination data for a selected examination centers of Anadolu University ODES was considered. However, because of large dimension of the problem, an exact solution could not be obtained by the mathematical model. Thus, this problem is solved by the proposed algorithm. Comparison of the results obtained by the multi objective heuristic algorithm and of the current state is given in Table IV. Results of multi-objective heuristic algorithm are better than those of the current situation for minimizing distance and booklet diversity. However, for maximum total examination building occupancy, multi-objective heuristic's performance is not as good as that of currently used schedule.

\section{Conclusions}

In this study, we have developed a new mathematical model for the multisession exams-building assignment for a real-life problem of the Anadolu University ODES that has 1400000 students. This model can not generate a solution because of the dimensions of the problem. In order solve the problem, we have developed a heuristic algorithm, known as multi-objective heuristic algorithm. When we compare the current situation to the results of multi-objective heuristic algorithm, we see that multiobjective heuristic algorithm gives better solution than the current solution for minimizing the distance and the booklet diversity. The most common way to solve this problem involves the use of heuristics and/or metaheuristics, that guide the search into promising areas of the space. Besides, based on the literature [13-15], hyperheuristics approaches, which may perform better than heuristics, can be considered as a future work. In addition, the invigilator-exam assignment problem can be considered, as a separate problem, in the future.

Anadolu University is a non-profit organization, so Anadolu University can bear the costs of the unused capacity of examination buildings. The mathematical model and multi-objective heuristic algorithm can provide a general solution approach for assigning students to examination buildings in educational institutions, which have same characteristics as Anadolu University ODES.

\section{References}

[1] Z. Kamisli Ozturk, G. Ozturk, M. Sagir, Int. J. Inform. Technol. Decision Making 9, 223 (2010).

[2] M.W. Carter, G. Laporte, S.Y. Leen, J. Operat. Res. Soc. 47, 373 (1996).

[3] M.W. Carter, J. Operations Res. 34, 193 (1986).

[4] A. Dammak, A. Elloum, H. Kamoun, Adv. Engin. Software 37, 659 (2006).

[5] P. Stefanov, A. Savic, G. Dobric, Acta Phys. Pol. A 128, B-138 (2015).

[6] F.H. Khan, U. Qamar, S. Bashir, Cognitive Computat. 8, 614 (2016).

[7] Q. Tang, Y. Shen, C. Hu, J. Zeng, W. Gong, Cognitive Computat. 5, 48 (2013).

[8] M.N.M Kahar, G. Kendall, Europ. J. Operat. Res. 207, 557 (2010).

[9] S.C Sarin, Y. Wang, A. Varadarajan, A.J. Sched, J. Schedul. 13, 131 (2010).

[10] M. Ayob, A. Malik, Int. J. Comput. Sci. Network Secur. 11, 187 (2011).

[11] H. Vermuyten, S. Lemmens, I. Marques, J. Belien, Europ. J. Operat. Res. 251, 651 (2016).

[12] Anadolu University, September 20, 2016, from www.anadolu.edu.tr/en/openeducation/examinations-2/examinations.

[13] F. Chen, L. Wang, B. Jiang, C. Wen, Cognitive Computat. 7, 464 (2015).

[14] J. Swan, J. Woodward, E. Özcan, G. Kendall, E. Burke, Conginitive Computat. 6, 66 (2014).

[15] J.C Ortiz-Bayliss, H. Terashima-Marín, S.E. ConantPablos, Conginitive Computat. 8, 429 (2016). 